\title{
Carnets
}

Revue électronique d'études françaises de l'APEF

Deuxième série - $16 \mid 2019$

Le Récit inachevé: études sur Mai 68

\section{Mai 1968 : La fête des Dieux}

Jean-Jacques Wunenburger

\section{Q OpenEdition}

\section{Journals}

\section{Édition électronique}

URL : http://journals.openedition.org/carnets/9625

DOI : 10.4000/carnets. 9625

ISSN : 1646-7698

Éditeur

APEF

\section{Référence électronique}

Jean-Jacques Wunenburger, «Mai 1968 : La fête des Dieux », Carnets [En ligne], Deuxième série - 16 | 2019, mis en ligne le 31 mai 2019, consulté le 09 novembre 2019. URL : http:// journals.openedition.org/carnets/9625; DOI : 10.4000/carnets.9625

Ce document a été généré automatiquement le 9 novembre 2019.

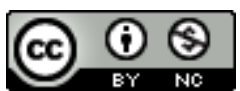

Carnets est mis à disposition selon les termes de la licence Creative Commons - Atribution - Pas d'utilisation commerciale 4.0 International. 


\title{
Mai 1968 : La fête des Dieux
}

\author{
Jean-Jacques Wunenburger
}

1 Les événements sociaux et politiques du printemps 1968 en France ont été une occasion assez rare d'assister à une effervescence collective, scandant des slogans porteurs de rêves et d'idéaux utopiques, sur un mode intempérant et intempestif, destinée à activer un changement brutal et massif des institutions et des mœurs. On peut, selon les angles d'approche et les systèmes d'interprétation, y voir tantôt une révolution destinée à accoucher à terme d'un monde nouveau, tantôt une révolte passagère et limitée contre un système appréhendé comme oppressif, tantôt une sorte d'inversion festive du monde au présent (dans le sillage des « fêtes des fous» médiévales et du carnaval). De même que ces évènements échappent à un concept unique et homogène, les images et les paroles qu'ils ont engendrées émargent dans différents registres idéologiques et symboliques, renvoyant à des filiations marxistes, anarchistes, mouvements alternatifs, contre-culture, etc. Cette pluralité bariolée de références et d'injonctions est de plus portée par des groupes aux identités variées et parfois incompatibles.

2 Loin de vouloir refaire l'histoire complexe, largement déjà restituée, de cette période, nous voudrions avant tout cerner l'imaginaire global de cette période, pour en dévoiler de grandes strates symboliques et mythiques. Cette "mythanalyse ", chère à Gilbert Durand, permettrait de voir dans cette période un moment significatif de flux et de confluence d'imaginaires antérieurs, sur fond d'une évolution selon des périodes longues, qui amènent à passer de la micro-histoire sociologique à la métahistoire du changement des grands récits.

De ce point de de vue, nous formulons l'hypothèse que les mythèmes dominants, revendiquant un nouveau monde ou un monde l'envers, se laissent interpréter comme la scénarisation et la célébration de deux divinités, Prométhée et Dionysos, en conflits mytho-culturels depuis longtemps, et qui se retrouvent sur la scène de l'histoire, mais selon des phases décalées. Mai 68 se laisserait donc comprendre à travers une typologie de divinités inspiratrices et selon un calendrier cyclique propre à chaque prototype. $\mathrm{Si}$ Prométhée, dieu de la modernité industrielle et du progrès, semble connaitre un ultime sursaut, Dionysos, emblème de la transgression pulsionnelle, paraît (re)prendre sa place par son irruption sauvage, avant que les deux ne quittent finalement l'histoire, à 
la fin $\mathrm{du} \mathrm{xx}^{\mathrm{e}}$ siècle, au profit d'un retour d'Hermès, figure des échanges et de la communication.

4 Autrement dit, le «moment 68 » peut se laisser analyser selon le modèle «potamologique » durandien, pour qui chaque imaginaire social est comme un fleuve qui connait successivement source, ruissellement, partage des eaux, confluence, dénomination, aménagement des rives et épuisement dans le delta ${ }^{1}$. Mais 68 pourrait même être compris comme une confluence de deux fleuves, un majeur et un mineur, ce qui provoque un tourbillon assez chaotique des flux, avant de donner naissance au fleuve post-68, une postmodernité communicationnelle. Comment décrire cette géographie et cette histoire des mythes dans les années 1960-1980, sur fond non d'une identité fermée unitaire, mais d'une bipolarité transitoire, médiatrice d'un monde nouveau en un sens différent de celui qui était fêté et prophétisé par les acteurs de mai 68 ?

\section{Les bassins fluviaux des imaginaires}

5 Les événements du printemps 68 apparaissent comme un moment de confluence de deux filiations mytho-idéologiques ${ }^{2}$, l'une liée au progressisme marxiste et à ses variations-déviations, l'autre à une contre-culture anarchico-gauchiste, qui prennent leurs sources, l'une plutôt en URSS, l'autre plutôt aux USA (et même en Californie).

\section{Le fleuve de la révolution prolétarienne marxiste-léniniste}

6 Si l'activisme contestataire du monde étudiant a été le plus réactif et le plus inventif en styles d'agitation et en proclamations libératrices, le mouvement de fond de la grève généralisée a été porté surtout par les syndicats et partis marxistes, qui ont cherché à récupérer le mouvement contestataire de la jeunesse («mouvement du 21 mars ») en le mettant au service des revendications des travailleurs. Mai 68 apparait bien, de ce point de vue, comme une phase un peu improvisée et opportuniste du récit marxiste de révolution prolétarienne contre l'ordre bourgeois, accaparant les instruments de production et exploitant la classe ouvrière. Cette version populaire s'enracine dans les analyses hégélo-marxiennes qui font de l'aliénation par le travail le moteur d'un renversement de la domination bourgeoise.

7 On a pu établir que ce grand récit du XIXe siècle de la révolution communiste, devait sa force de conviction et de contagion au renforcement par le mythe latent de la souffrance rédemptrice du récit chrétien, le Christ symbolisant l'aliénation de l'humanité adamique condamnée au travail après la Chute, avant de connaitre un salut libérateur ${ }^{3}$. Cette matrice théologico-politique de la révolution prolétarienne a été renforcée par la tradition apocalyptique millénariste, depuis les prophéties médiévales de Joachin de flore, pour qui la violence des justiciers de Dieu devait hâter le temps du Troisième Âge, phase d'un millenium de paix et de justice ${ }^{4}$.

8 Mais à l'intérieur de ce grand récit, entretenu internationalement par l'URSS, se sont mis à proliférer plusieurs micro-récits, qui en sont autant de variations; on peut en identifier quelques familles :

a. l'orthodoxie communiste, contrôlée par le parti communiste, inféodé à Moscou, qui repose sur une organisation centralisée, hiérarchique, bureaucratique, qui va de plus en plus apparaitre comme une version du totalitarisme ${ }^{5}$; 
b. le trotskisme, qui repose sur des organisations quasi secrètes, sectaires et cloisonnées, pour subvertir le système de l'intérieur (la taupe) et parfois armées, pour faire régner la violence contre les ennemis présumés que sont les agents et les organisations du capitalisme ;

c. une extrême gauche prolétarienne, qui cherche ses sources d'inspiration dans le maoïsme ;

d. un gauchisme spontanéiste, plus activiste que pratiquant l'entrisme, mais qui était déjà taxé de « maladie infantile du communisme » par Lénine ;

e. un courant autogestionnaire, d'inspiration Proudhonienne, nourrie de l'expérience titiste en Yougoslavie, et qui inspirera des activismes sociaux après la période 68 (années 70);

f. en marge, un mouvement dissident plus hégélien que marxien, dit situationniste, qui se déploie selon des références plus ludiques qu'organisationnelles, dont les analyses rejoignent déjà les rives de l'imaginaire du second fleuve.

\section{Le fleuve de la contre-culture américaine}

Paradoxalement, une autre racine contestataire, largement présente dans les motivations et revendications des étudiants, ne vient pas d'URSS mais des USA, en particulier des universités californiennes (Berkeley). Elle s'inscrit dans un autre grand récit spontanéiste alternatif de transgression des normes et des limites imposées par la culture occidentale, plus que par la seule classe bourgeoise («il est interdit d'interdire ", "sous les pavés la plage ", «l'imagination au pouvoir »). Cet imaginaire (déjà préfiguré par les analyses de l'Ecole de Francfort ${ }^{6}$ ) est enfant d'Orphée, de la sexualité libérée, de la nudité, du désir, de la communauté, etc. Il combat la morale bourgeoise, de la loi, de la norme, comme le récit marxiste combat l'accumulation et l'exploitation, mais en dénonçant à son opposé l'aliénation même du travail et de ses configurations culturelles rationnelles. Cet imaginaire d'une jeunesse non salariée, aux antipodes des intérêts de la classe ouvrière, se sent plus proche de Dionysos ${ }^{7}$ que de Prométhée et capte des mythèmes du paganisme (antichrétien) et des hérésies gnostiques (adamites) ${ }^{8}$. L'imaginaire hédoniste et festif trouve ses expressions les plus récentes dans le mouvement hippie et aspire davantage à la réalisation d'un âge d'or naturaliste qu'à l'avènement d'une société industrielle libérée de l'exploitation.

\section{Quelques problèmes épistémologiques des courants d'imaginaire}

10 La mythanalyse durandienne en assimilant chaque type d'imaginaire à l'écoulement d'un fleuve en suivant des phases cruciales, permet de mieux comprendre la dynamique des formes et forces symboliques, mais aussi de suivre la dualité de deux ou plusieurs imaginaires socio-politiques et donc leurs phasages, leurs rencontres, leurs tendances à s'immiscer, à se mélanger, selon leurs tensions internes. On peut dès lors dégager quelques questions clés pour enrichir l'analyse de la période, loin des stéréotypes essentialistes et hâtivement monovalents.

11 Loin de correspondre à un imaginaire unique, l'effervescence festive du printemps 68 prend appui sur deux visions et sémantiques différentes voire opposées, l'une issue de l'histoire du travail industriel et de la lutte des classes, au sens marxiste, l'autre, d'une contestation de la culture moderne capitaliste (travail, réussite, enrichissement), au profit d'une socialité douce, communautaire, esthétique, fondé sur la jouissance plus que sur le travail et ses sublimations. Mais ces deux récits, avec leurs mythèmes 
propres, ont du mal à converger, travail et loisir, volonté libératrice et désir hédoniste se révélant à bien des égards incompatibles. Il reste quelques tentatives minoritaires d'hybridation et de syncrétisme: le freudo-marxisme (W. Reich), entre marxisme et psychanalyse, veut intégrer la libération sexuelle dans le scénario de la libération des travailleurs; le situationnisme (R. Vaneighem, 1992 ; G. Debord, 1996), qui dénonce la société du spectacle, sous-produit de la société de consommation (J. Baudrillard) comme fausse vie, tout en récupérant les idéologèmes de Hegel et Marx déjà mis en avant par l'Ecole de Francfort (réification).

12 Le mythème du rassemblement fusionnel des catégories sociales (jeunesse et classe ouvrière) intègre la dimension de la fête et du festif. Sans être pacifistes, les mouvements contestataires ne recourent pas une violence aveugle et illimitée (attentats, terrorisme, prise d'otage), mais plutôt à une grève, au blocage, à la suspension du travail rendant possible des rencontres collectives, joyeuses, à dominante anarchistes. Par-là les agitateurs de mai 68 retrouvent la culture symbolique de la fête, qui était une forme de rupture de l'espace et du temps social, un moyen alternatif d'inverser l'ordre. Mais le dyonysisme latent se réduit souvent à une pure expérience de la transgression, un vécu sans lien avec une institution rituelle qui codifiait le temps de la fête et imposait des figures mythiques traditionnelles. La fête devient ainsi plus une forme de socialité anomique, hors norme, que la recherche d'une régulation symbolique d'un ordre, qui est toujours dans les sociétés anciennes rétabli. On est plus dans la dérive d'un mythe tronqué, dans le fantasme d'une fête permanente que dans l'adhésion à une culture de l'inversion, inscrite dans un récit symbolique ${ }^{9}$. Ce traitement mythique régressif n'est-il pas déjà une des sources de sa propre caducité et de son impuissance, comme le montre la lente usure du mythe 68 dans les décades suivantes?

13 Les formes d'expression ludiques les plus pratiquées se rapprochent de pratiques artistiques (depuis le street art jusqu'à l'improvisation musicale et le théâtre du happening). Loin de passer par une vraie violence destructrice de l'ordre établi ni par un bouleversement des institutions, qui n'ont guère été ébranlées, la fête 68 décompose et recompose surtout les pratiques artistiques, en les émancipant des normes. L'art devient politique et le politique devient manifestation artistique, l'un et l'autre devant revendiquer, expérimenter et célébrer des valeurs autres que la loi et l'ordre bourgeois. L'époque voit ainsi se développer un ensemble de pratiques nouvelles (happening, performance, living theater), qui resteront longtemps après des formes pour faire de l'art un moyen d'engagement politique voire de contestation politique de la société. Mais en s'esthétisant, la contestation rompt de plus en plus avec le flux marxiste, qui compte avant tout sur le changement économique de la société pour faire advenir une société nouvelle. L'imaginaire instantanéiste du jeu prend la place de l'imaginaire du futur, centré sur un travail libéré.

14 Le caractère festif a souvent été couplé avec celui de l'utopie. Cette catégorie connaît à cette époque une évolution sémantique importante mais très chargé d'approximations. L'air du temps se ressent de l'ambivalence du vocabulaire établi, car tantôt l'utopie est considérée comme une idéalité anhistorique à dominante conservatrice qui inhiberait une vraie révolution (K. Mannheim, 1956), tantôt elle est au contraire le moteur de la révolution (E. Bloch, 1996). Même dans ce dernier cas, elle est supplantée par la catégorie de la fête, qui peut être assimilée à une utopie inscrite dans l'ici et le maintenant, vidant ainsi la matrice utopique de son caractère de futur plus ou moins 
réalisable. L'utopisme, avec sa charge émotionnelle d'espérance, se trouve donc en porte à faux avec une sémantique tantôt positive tantôt négative, empêchant ainsi de nommer vraiment les évènements.

Enfin la coexistence et la simultanéité du marxisme et de l'anarchisme peuvent inciter à penser à leur égale force d'idéation et d'emprise imaginaire. Or la mythanalyse durandienne permettrait de penser un phasage différencié de leur maturation ou déclin. Si Gilbert Durand a moins étudié cette synchronisation de plusieurs fleuves d'imaginaires, il a théorisé les caractères récessifs et dominant de chacune des mythologies, sur le modèle de l'actualisation et de la potentialisation du logicien Stéphane Lupasco (2003), ce qui présuppose un moment d'acmè, d'égal tropisme, un moment nommé « état $\mathrm{T}$ ». Il resterait à savoir si Mai 68 pourrait être compris comme ce moment d'équipotence ou déjà comme une rupture, chaque étiage du fleuve suivant une tendance propre. On pourrait peut-être dater de mai 68 le début -inattendu et imperceptible - du reflux du fleuve marxiste, arrivé à son point de renversement (Monnerot, 1970), ce que l'histoire entérinera avec la chute de l'URSS, et comme l'actualisation de forces dionysiaques, qui vont se déplacer par la suite dans le caractère orgiastique de la société de consommation, avant d'être déterritorialisées et virtualisées dans la société de communication, qui signe l'arrivée d'Hermès (Monnerot, 1970 ; McLuhan, 2017).

\section{Quelques controverses}

16 Au-delà des événements et des expériences, mai 68 a nourri des jugements, évaluations et critiques très vives, tranchées, passionnées, au centre d'innombrables débats. La chaleur et l'intensité des mouvements avec leurs conséquences particulières (grèves), ont animé et amplifié les positions intellectuelles et idéologiques: d'un côté le marxisme orthodoxe a connu d'innombrables remises en cause internes, aboutissant à des fractionnements et dissidences. Le climat sera propice à la naissance de l'antitotalitarisme qui va croitre jusqu'au moment Soljnenytsine (Bernard-Henri Lévy, 1977 ; André Glucksmann, 1977). De l'autre côté, l'inspiration de la contre-culture a aussi bien encouragé à la critique du marxisme qu'à son contournement radical au profit d'une altercation propre à la contre-culture, qui va servir de prélude à l'émergence de nouveaux flux d'imaginaires: la croissance d'idéaux écologiques, adversaires de la consommation, ancêtres de la décroissance; l'entrée dans une ère libertaire technophile, dont Internet et l'informatique en réseaux horizontaux seront les bénéficiaires.

17 Michel Maffesoli a développé dans les années 1980 une sociologie de la vie quotidienne, en phase avec les tendances profondes de l'esprit post-moderne. Il vise à interpréter la socialité post-68 comme une régénération par la reliance, la circulation de la parole et de la sexualité, le besoin de communauté, l'exaltation du présent, le goût des transgressions. Ces signes nouveaux marqueraient la fin de l'énergétisme et du productivisme imposés par la société industrielle, placée quant à elle sous le signe de Prométhée. L'hypothèse d'un retour du dionysiaque dans des sociétés marquées par l'hypertrophie du politique, du travail, de la loi, de la morale se trouverait corroborée par l'orgiasme multiforme qui conduit les individus à fusionner dans des expériences groupales, à intensifier leurs émotions, en particulier sexuelles, à jouer avec la vie et la mort sur fond d'un tragique esthétisé. 
18 A l'inverse pour Jean Brun, la transgression dionysiaque correspond certes à une aspiration contemporaine de la société et de la culture à abolir les limites du Moi, à cultiver une frénésie des sens et de l'esprit, mais loin de conduire à une sorte de régénération sociale, elle ne serait qu'un facteur de déstructuration psychologique, une folie sociale, qui trouve ses racines cachées dans un désir métaphysique de changer la condition humaine. Ainsi Jean Brun voit dans le dieu de l'extase la figure de la frénésie généralisée dans la culture contemporaine. Celle-ci n'inspire pas seulement les comportements quotidiens à travers les multiples excitations engendrées par les techniques (vitesse, bruit, etc.) ou les fascinations idéologiques pour les fureurs de la Révolution politique, mais aussi les pratiques intellectuelles des scientifiques et philosophes qui attendent des constructions conceptuelles (évolutionnisme, structuralisme, etc.) un véritable salut métaphysique. Autrement dit, le désir dionysiaque s'exprimerait non seulement dans la sexualité et la socialité mais aussi dans les vertiges spéculatifs ou les expérimentations artistiques (celles du happening, en particulier). A travers Dionysos les hommes chercheraient, sous de nouvelles formes, une ivresse de la transgression, condition d'une émancipation de leur finitude ${ }^{10}$.

Quant à Prométhée, il va connaitre en un sens son propre retrait, sur fond d'une société où la consommation va prendre le relais de la production, le travail sera dévalué par rapport aux loisirs de masse (devancé par le mythe solaire du mythique "Club Méditerranée »). Mais la victoire historique du capitalisme sur le communisme après la chute de l'URSS va en quelque sorte faire se rejoindre momentanément le travail, source d'épanouissement par la performance et les gains, et la consommation, dans le cadre d'une économie globalisée où dominent industries de l'imaginaire (block booster hollywoodiens, jeux vidéo) et de la sexualité (pornographie).

\section{Le devenir de la fête des dieux}

20 Le printemps 68 a représenté un sommet flamboyant d'une forme de civilisation moderne, mais son intensité, sa rapidité n'ont pas vraiment donné de suite ni fait école. Les deux dieux au rendez-vous de l'histoire ont connu en un sens leur acmé avant de faire place à un nouveau fleuve.

21 Le marxisme, malgré sa quasi divinisation, n'a jamais repris son ascendant antérieur et va suivre la lente érosion des idées socialo-communistes. Malgré des sursauts violents (terrorisme d'extrême gauche avec la "Bande à Baader" en Allemagne, Brigades rouges en Italie), le marxisme-léninisme et surtout maoïste va perdre de son emprise, surtout après les bilans du totalitarisme chinois ou cambodgien, qui aggravent le soviétique.

Quant à l'imaginaire dionysiaque, malgré sa greffe symbolique sur d'autres courants, il a été phagocyté par un dyonysisme de la marchandise et de la consommation, perdant ainsi son potentiel d'altercation. Progressivement, la contre-culture hippie californienne et ses mimétismes européens ont été absorbés par Hermès, dieu de la communication. Les outils informatiques développés par la Silicon Valley sont apparus comme des moyens d'étendre l'individualisme libertaire, à l'insu souvent des systèmes cachés de contrôle, avant que ceux-ci ne prennent réellement le contrôle via les GAFA. 
ans après, quels sont les nouveaux imaginaires? Prométhée est devenu via les technologies d'information, une forme du nouveau Dédale, un avatar du Golem avec la mythologie du robot et de la robotisation de la vie (Trans-humanisme). Le travail a été remplacé par les logiciels et les algorithmes, qui constituent les bras armés de la société de consumation globalisée, au risque de la dégradation de l'environnement naturel. Dionysos, comme célébration du désir et du sexe, a cédé la place à Gaia, une déesse animiste de la nature et du cosmos, qui invite les humains à fusionner avec elle en renonçant aux désirs corrupteurs et insatiables, au profit de désirs conformes à l'ordre naturel sacré et inviolable. Revient alors le mythe de l'âge d'or avec une humanité dispensée du travail, menant une vie paisible en compagnie d'animaux plus sages que les humains.

Finalement, les dieux de mai 68 ont déserté l'histoire mais nous sommes à nouveau au confluent d'autres fleuves, celui de la « démiurgie » qui remplace le vivant et celui de la "phyturgie", qui se règle sur la spontanéité de la vie, animale (animalisme et antispécisme) et même végétale (avec le culte de l'arbre). Notre avenir est placé sous les figures d'autres divinités, à nouveau en opposition, qui vont déployer des imaginaires antinomiques de la robotique (devancée par la science-fiction) et de l'animalisme (l'humain cherchant à rejoindre le monde animal dont l'émancipation aurait entrainé une catastrophe). Mai 68 apparait ainsi comme un moment anachronique, archaïque de nos imaginaires contemporains, mais qui en un sens représente peut-être le feu d'artifices tardif d'un monde du 19es siècle, pour l'instant totalement dépassé, mais qui fera sans doute un retour sous d'autres visages, un jour.

\section{BIBLIOGRAPHIE}

ARENDT, Hannah (2005). Le Système totalitaire. Paris : Gallimard.

DURAND, Gilbert (1996). Introduction à la mythodologie, mythes et sociétés. Paris : Albin Michel.

BELINI, Paolo (2011). Mythopies techno-politiques : Etat-nation, empire et globalisation, Sesto San Giovanni : Mimesis.

BLOCH, Ernst (1996). L'Esprit de l'utopie. Paris : Gallimard.

COHN, Norman (1983). Les Fanatiques de l'apocalypse. Paris : Payot.

DEBORD, Guy (1996). La Société du spectacle. Paris : Gallimard, « Folio ».

ELIADE, Mircea (1949). Le Mythe de l'éternel retour. Paris : Gallimard.

GLUCKSMANN, André (1977). La Cuisinière et le mangeur d'hommes. Paris : Seuil.

LACARRIÈRE, Jacques (1994). Les Gnostiques. Paris : Albin Michel.

LÉVY, Bernard-Henri (1977). La Barbarie à visage humain. Paris : Grasset.

LUBAC, Henri de (2014). La Postérité spirituelle de Joachim de Flore. Paris : Cerf.

LUPASCO, Stéphane (2003). Les Trois Matières. Strasbourg : Sophon Éditions.

Carnets, Deuxième série - 16 | 2019 
MANNHEIM, Karl (1956) Idéologie et utopie. Paris : Librairie Marcel Rivière et $C^{\text {ie }}$.

MARCUSE, Herbert (1968). L'Homme unidimensionnel. Paris : Minuit.

MCLUHAN, Herbert Marshall (2017). La Galaxie Gutemberg. Paris : CNRS.

MONNEROT, Jules (1970). Sociologie de la révolution. Paris : Fayard.

MONNEYRON, Frédéric ; XIBERRAS, Martine (2008). Le Monde hippie : De l'imaginaire psychédélique à

la révolution informatique. Paris : Imago.

NIETZSCHE, Friedrich (2015). La Naissance de la tragédie grecque. Paris : Garnier-Flammarion.

SIRONNEAU, Jean-Pierre (2000). Figures de l'imaginaire religieux et dérives idéologiques. Paris :

L'Harmattan.

VANEIGHEM, Raoul (1992). Traité de savoir-vivre à l'usage des jeunes générations. Paris : Gallimard, «Folio ».

WUNENBURGER, Jean-Jacques (1977). La Fête, le jeu et le sacré. Paris : Éditions Universitaires.

WUNENBURGER, Jean-Jacques (1979). L'Utopie ou la crise de l'imaginaire. Paris : Jean-Pierre Éditeur.

WUNENBURGER, Jean-Jacques (2000). « Figures de Dionysos : renouveau et obstacles herméneutiques contemporains » in Ilana Zinguer (éd.). Dionysos, origines et résurgences. Paris : Vrin, pp. 41-48.

\section{NOTES}

1. Voir Gilbert Durand (1996).

2. On gagnerait à affiner les catégories des représentations de l'imaginaire comme F. Araujo qui parle d' « idéologème » ou de P. Bellini qui distingue des « mythopies (P. Bellini, 2011).

3. Voir M. Eliade (1949) et J.-P Sironneau (2000).

4. Voir N. Cohn (1983), H de Lubac (2014) et J.-J. Wunenburger (1979).

5. H. Arendt (2005).

6. Ce mouvement à la suite de Adorno, autour de Horkheimer, Habermas, Marcuse, etc., critique plus largement que le marxisme non seulement les instruments de production mais une rationalité réificatrice de la vie. Voir $\mathrm{H}$. Marcuse (1968) qui célèbre Orphée.

7. Dionysos fut déjà célébré par F. Nietzsche (2015) comme la figure de la vie irrépressible contre l'ordre rationnel d'Apollon.

8. Voir J. Lacarrière (1994), F. Monneyron et M. Xiberras (2008).

9. Nous avons développé ce thème en 1977 (J.-J- Wunnenburger, 1977).

10. Nous avons développé ce thème dans «Figures de Dionysos: renouveau et obstacles herméneutiques contemporains » (Wunnenburger, 2000).

\section{RÉSUMÉS}

L'effervescence d'idées et d'images des mouvements sociaux de mai 68, surtout vus de France, loin d'être monolithiques, se compose de deux grandes thématiques mythiques: les unes, 
prennent leur source dans divers marxismes révolutionnaires, incluant le prolétariat en tension avec le mouvement intellectuel étudiant, et les autres, qui puisent dans divers courants anarchistes et gnostiques, aspirant à une société libertaire.

On examine ces courants et leurs imaginaires à travers le modèle métaphorique de Gilbert Durand, qui assimile les différentes phases d'un imaginaire collectif aux étapes de la morphologie d'un fleuve. Il apparait ainsi que ces imaginaires, en grande partie antithétiques, remontent aux grandes mythologies des $\mathrm{XIX}^{\mathrm{e}}$ et $\mathrm{XX}^{\mathrm{e}}$ siècles, ce qui expliquerait qu'ils n'aient pas eu de véritable postérité, laissant place à d'autres imaginaires contemporains.

The effervescence of ideas and images of May 68 social movements - above all approached from a French point of view, and, by no means, monolithic - consists of two major mythic topics. Some of them come from different revolutionary Marxisms, including the proletariat in tension with students' intellectual movement, and the other ones tap into anarchist and gnostic mainstreams that long for a libertarian society. We will study these mainstreams and their imageries through Gilbert Durand's metaphorical model, which liken the different phases of a collective imagery to the steps of a river morphology. It appears that these imageries, most of them antithetic, date back to the XIX and xxth centuries, which explains the lack of any genuine posterity, and leave room to other contemporary imageries.

\section{INDEX}

Keywords : celebration, Marxism, anarchism, imagination, freedom, revolution, divine archetypes

Mots-clés : fête, marxisme, anarchisme, imagination, liberté, révolution, archétypes divins

\section{AUTEUR}

\section{JEAN-JACQUES WUNENBURGER}

Institut de Recherches Philosophiques de Lyon

jean-jacques.wunenburger[at]wanadoo.fr 